\title{
Physical Scarcity of Water in a Region
}

\author{
Yu Zhang \\ School of North China Electricity Power University, Baoding 071000, China
}

Keywords: Logistic Model, s-type Curve, ARMA Model, Grey Relational.

\begin{abstract}
This report builds a s-type curve model which defines the change of per capita water consumption following the change of GDP, and it defines the rate of per capita satisfaction of using water which is considered as the index to judge the ability of the region to provide clean water to meet the needs of its population. Under certain prediction, analyzing and function fitting, we can achieve to get the rate of per capita satisfaction of using water in the future by predicting rainfall capacity to determine other water resources.
\end{abstract}

\section{Introduction}

We build a general model to provide a measurement of the ability of a region to provide clean water to meet the needs of its population. Considering the ability of the region to provide clean water, it should satisfy the balance of both supply and demand in the region. In the condition that all the population are using clean water; per capita household water occupancy volume and the number of population both determine the ability of a region to provide clean water to meet the needs of its population. We need to define the rate of per capita satisfaction of using water to reflect the ability of a region to provide clean water to meet the needs of its population, the s-type curve of per capita water consumption and the occupancy rate of per-capita water consumption. The rate of per capita satisfaction of water consumption is that per capita water consumption with its ratio of per capita water consumption function with the region society condition. The bigger the rate of per capita satisfaction of using water is, the stronger the ability of a region to provide clean water to meet the needs of its population is. We can get per capita water consumption on arbitrary time and space by historical data. Per capita water consumption divides by the stable value at the s-type curve of the per capita water consumption to express the ability of a region to provide clean water to meet the needs of its population at the time in this region.

We need to get total water consumption by historical data including surface water supply volume, underground water supply volume, other water supply volume, quantity of water diversion, water storage and so on. In the condition that people are not establishing new water conservancy project and not overly exploring, the ability of water storage, so the water-regulating capacity and the source of underground water are constant. Therefore, the dynamic change of the water supply quantity in the region are caused by the dynamic change of surface water resources and other water resources. We predict total water supply quantity and the occupancy rate of per-capita using water in the future several years by the time series prediction method. By predicting the number of people in the future several years by Logistic Model, we can Get per capita water consumption in the future several years by these prediction. We could also determine the ability of a region to provide clean water to meet the needs of its population in the future.

\section{Model Building}

The definition of the degree of per capita satisfaction of using water reflects a measure of the ability of a region to provide clean water to meet the needs of its population which is the per capita water consumption with its ratio of per capita water consumption function with the society condition in the region ${ }^{[3]}$. The per capita water consumption is equal to the region available clean water with its ratio of the number of population.

For the historical time, we can directly substitute into historical data and indicate stable value at the s-type curve of the per capita water consumption by finding s-type curve of per capita water 
consumption function with the changing of GDP. These two values divide by each other, so we can determine the ability of a region to provide clean water to meet the needs of its population at the time in the region. The introduction of s-type curve is shown as Fig.2-1

By comparing different period in inland and aboard, and different size of per capita water consumption in different region. The factors that influences per capita water consumption are natural conditions constraint, social system, idea restriction and social economic development level. Under different natural condition, different social system, different notion and different social economic development level. The relationship between per capita water consumption and per capita GDP can be described as s-type curve ${ }^{[1]}$. This progress is shown as below:

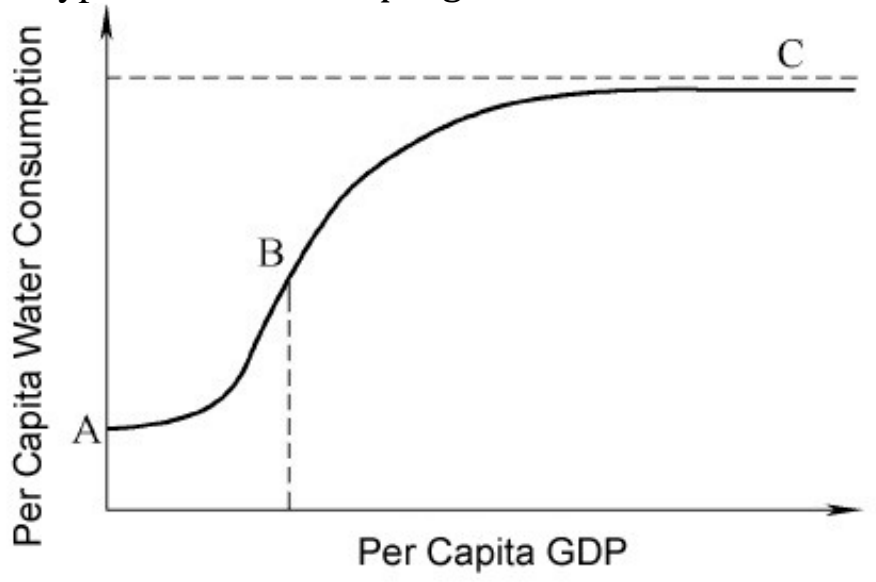

Figure 2-1 The signal of s-type curve

In the Figure 2-1 there are three pivotal points: point $A$ is life water consumption when per capita is approaching to zero; point $B$ is that the per capita water consumption will increase following the enhancement of per capita GDP which is the curve inflection point .Before the point $\mathrm{B}$, the acceleration is indicating bigger and bigger. After this point, the acceleration is becoming smaller. Point $C$ is the extreme point in the increasing curve rather than a fixed point.

According to the character of the Figure 2-1 we choose act tangent function to do fitting and indicate the function of the curve:

$$
Q D W=a \tan ^{-1}(G D P-b)+c
$$

In the function $Q D W$ is per capita water consumption $\{L /($ person $\cdot d)\} ; G D P$ is per capita $\operatorname{GDP}\left(R M B 10^{4} /\right.$ person $)$.

Firstly we choose the value of $\mathrm{b}$, then calculate the GDP corresponding $\tan ^{-1}(G D P-b)$ in different period and command $x=\tan ^{-1}(G D P-b), y=Q D W$. In this condition the function can be changed to solve a and $c$, the function is shown as below:

$$
\begin{gathered}
\mathrm{a}=\frac{n \sum_{i=1}^{n} x_{i} y_{i}-\sum_{i=1}^{n} x_{i} \sum_{i=1}^{n} y_{i}}{n \sum_{i=1}^{n} x_{i}^{2}-\left(\sum_{i=1}^{n} x_{i}\right)^{2}} \\
\mathrm{c}=\frac{1}{n} \sum_{i=1}^{n} y_{i}-\frac{a}{n} \sum_{i=1}^{n} x_{i}
\end{gathered}
$$

In the function $\mathrm{i}$ is number of the initial data column $(x, y)$; $\mathrm{n}$ is the total number of the data column.

At last the judge of the fitting generally uses mapping to judge if the curve can represent the trend of initial data; we can also calculate the correlation coefficient $\mathrm{R}$ and judge the fitting according to it generally requesting $R \geq 0.8$. If the fitting is not good,we need change $b$ and repeat this step and compare.

\section{Measure of the Ability of a Region}

For the future, we use prediction method to get a measure of the ability of a region to provide clean water to meet the needs of its population. The specific implement way is shown as below: 
1) The prediction for the number of population

We use the Logistic Model, because the number of population will get impeded by natural sources and environmental conditions. It makes the number of population decrease after it increased to a certain number. The prediction of the number of population in this model is:

$$
x(t)=\frac{x_{m}}{1+\left(\frac{x_{m}}{x_{0}}-1\right) e^{-r t}}
$$

$x(t)$ is the number of people at $\mathrm{t}, x_{m}$ is the maximum number of people which the natural sources and environmental conditions can be included, $x_{0}$ is the number of people at the initial time, $r$ is the degree of population growth.

2) The prediction for precipitation

We use ARMA time series model. For the general stable array $\left\{X_{t}, t=0, \pm 1, \pm 2, \cdots\right\}$, the mean of it is assumed $\mathrm{E}\left(X_{t}\right)=\mu$ and satisfy the below model:

$$
\begin{gathered}
\left(X_{t}-\mu\right)-\varphi_{1}\left(X_{t-1}-\mu\right)-\cdots-\varphi_{p}\left(X_{t-p}-\mu\right) \\
=\varepsilon_{t}-\theta_{1} \varepsilon_{t-1}-\cdots-\theta_{q} \varepsilon_{t-q}
\end{gathered}
$$

Actually: $\varepsilon_{t}$ is stationary white noise which the mean is zero and the variance is $\sigma_{\varepsilon}^{2}$. We call $X_{t}$ is autoregressive-moving average sequence which the order is p and q, abbrevd ARMA (p, q) sequence. Using the after shift operator which is $\varphi(B)$ and $\theta(B)$ the Function (5) could be represented :

$$
\varphi(B)\left(X_{t}-\mu\right)=\theta(B) \varepsilon_{t}
$$

The order selection of ARMA model uses AIC criterion and choose $\mathrm{p}$ and $\mathrm{q}$ to make

$$
\min A I C=n \ln \hat{\sigma}_{\epsilon}^{2}+2(p+q+1)
$$

In the function, $n$ is the sample size; $\hat{\sigma}_{\epsilon}^{2}$ is the estimate of $\sigma_{\varepsilon}^{2}$. If when $p=\hat{p}, q=\hat{q}$, the function reaches to minimum and is thought array is $\operatorname{ARMA}(\hat{p}, \hat{q})$.

The chi-square test of ARMA model:

The residual of the fitting model is assumed to $\hat{\varepsilon}_{t}$ which is the estimate of $\varepsilon_{t}$.For example, for AR (p) we assume the estimates of unknown parameters are $\hat{\varphi}_{1}, \hat{\varphi}_{2}, \cdots, \hat{\varphi}_{p}$. And the residual is:

$$
\hat{\varepsilon}_{t}=X_{t}-\hat{\varphi}_{1} X_{t-1}-\cdots-\hat{\varphi}_{p} X_{t-p}, \mathrm{t}=1,2, \cdots, \mathrm{n}
$$

In this function we assume $X_{0}=X_{-1}=\cdots=X_{1-p}=0$.

Make:

$$
\eta_{k}=\frac{\sum_{i=1}^{n-k} \hat{\varepsilon}_{t} \hat{\varepsilon}_{t+k}}{\sum_{i=1}^{n} \hat{\varepsilon}_{t}^{2}}, k=1,2, \cdots, L
$$

Among it $L$ is the tailing number of autocorrelation coefficient $\hat{\varepsilon}_{t}$. The $\chi^{2}$ test statistic of Ljung-Box is:

$$
\chi^{2}=n(n+2) \sum_{k=1}^{L} \frac{\eta_{k}^{2}}{n-k}
$$

The assumption of the test is:

$H_{0}: \rho_{k}=0$, when $k \leq L ; H_{1}$ : for some $k \leq L, \rho_{k} \neq 0$.

When the assumption of $H_{0}$ establishe, if sample size $\mathrm{n}$ is enough big, $\chi^{2}$ is likely $\chi^{2}(L-r)$ distribution, $r$ is the number of model parameters.

The way of $\chi^{2}$ test: we give the significant nature $\alpha$, searching table gets the quantile $\chi_{\alpha}^{2}(L-r)$ is above $\alpha$. So when $\chi^{2}>\chi_{\alpha}^{2}(L), H_{0}$ is refused and we can think $\varepsilon_{t}$ is not the white noise and the model does not pass the test; but when $\chi^{2} \leq \chi_{\alpha}^{2}(L), H_{0}$ is accepted and we can think $\varepsilon_{t}$ is the white noise and the model pass the test.

The prediction of $\operatorname{ARMA}(p, q)$ array:

The $\mathrm{m}$ step prediction of time series array is according to the value of $\left\{X_{k}, X_{k-1}, \cdots\right\}$ to estimate the random variable on future $k+m$ time. The estimate is denoted by $\hat{X}_{k}(m)$ which is the liner combination of $X_{k}, X_{k-1}, \cdots$.

For $\operatorname{ARMA}(\mathrm{p}, \mathrm{q})$ sequence, there is:

$$
\hat{X}_{k}(m)=\varphi_{1} \hat{X}_{k}(m-1)+\varphi_{2} \hat{X}_{k}(m-2)+\cdots+\varphi_{p} \hat{X}_{k}(m-p), m>p
$$


So we only need to know $\hat{X}_{k}(1), \hat{X}_{k}(2), \cdots, \hat{X}_{k}(p)$ and can calculate $\hat{X}_{k}(m), m>p$ by recursiving. We need to define prediction vector

Letbe:

$$
\hat{X}_{k}(q)=\left[\hat{X}_{k}(1), \hat{X}_{k}(2), \cdots, \hat{X}_{k}(q)\right]^{T}
$$

$$
\varphi_{j}^{*}=\left\{\begin{array}{l}
\varphi_{j}, j=1,2, \cdots, p, \\
0, j>p .
\end{array}\right.
$$

We can get the below recursuving function:

$$
\hat{X}_{k+1}^{(q)}=\left[\begin{array}{ccccc}
-G_{1} & 1 & 0 & \cdots & 0 \\
-G_{2} & 0 & 1 & \cdots & 0 \\
\vdots & \vdots & \vdots & \ddots & \vdots \\
-G_{q-1} & 0 & 0 & \cdots & 1 \\
-G_{q}+\varphi_{q}^{*} \varphi_{q-1}^{*} \varphi_{q-2}^{*} & \cdots & \varphi_{1}^{*}
\end{array}\right] \hat{X}_{k}^{(q)}+\left[\begin{array}{c}
G_{1} \\
G_{2} \\
\vdots \\
G_{q-1} \\
G_{q}
\end{array}\right] X_{k+1}+\left[\begin{array}{c}
0 \\
0 \\
\vdots \\
0 \\
\sum_{j=q+1} \varphi_{j}^{*} X_{k+q+1-j}
\end{array}\right]
$$

3) Grey relational grade analysis

Grey relational grade analysis is according to the similarity degree of curve set shape to judge if the connection is close. The closer the curves are, the larger the connection of corresponding sequence is. On the contrary the connection is smaller.

Doing grey relational grade analysis firstly need unitary data. We assume the reference number series after unitary data is:

$$
X_{0}^{\prime}=\left\{x_{0}^{\prime}(1), x_{0}^{\prime}(2), \cdots, x_{0}^{\prime}(n)\right\}
$$

The comparable sequence is;

$$
\begin{gathered}
X_{i}^{\prime}=\left\{x_{i}^{\prime}(1), x_{i}^{\prime}(2), \cdots, x_{i}^{\prime}(n)\right\}, \quad i-1,2, \cdots, m \\
\Delta_{i}(k)=\left|x_{0}^{\prime}(k)-x_{i}^{\prime}(k)\right|, \quad k=1,2, \cdots n
\end{gathered}
$$

The maximum and minimum difference between bipolar are:

$$
\begin{aligned}
& \Delta(\max )=\max _{i} \max _{k} \Delta_{i}(k) \\
& \Delta(\min )=\min _{i} \min _{k} \Delta_{i}(k)
\end{aligned}
$$

The correlation coefficient is

$$
\gamma_{0 i}(k)=\frac{\Delta(\min )+\rho \Delta(\max )}{\Delta_{i}(k)+\rho \Delta(\max )}, \quad \rho \epsilon(0,1), k=1,2, \cdots, m
$$

4) Predict using fitting

We fit and analyze for some data which the changing trend is more smooth to predict and analyze in the future by fitting curve.

5) A measure of the ability of a region to provide clean water to meet the needs of its population

We get the future population total water consumption by predicting future total water requirement and population water utilization. And get per capita water consumption which is the future population total water consumption with ratio of the number of population ${ }^{[2]}$ in the future. And get the degree of satisfaction of per capita using water $M$ which is the per capita water consumption with ratio of the stable value in s-type in the region.

\section{Summary}

To get the rate of per capita satisfaction of using water in the future, we build the Logistic model to predict the number of population in the future. We also establish the ARMA model to predict rainfall capacity in the future and determine the relationship between rainfall capacity and all types of water resources by analyzing grey relational. Then we will determine the certain function by fitting.

\section{References}

[1]. ZUO Qi-ting. Interval S model for forecasting water consumption per capita. Journal of Hydraulic Engineering.2008 NO.3,p.351-354. 
[2]. Zeng Fashen .Analysis and optimal allocation of water resources supply and demand in Xi'an City.2008,p.13-15

[3]. Cao liqin. Analysis of hydrological characteristics of Zhengzhou City. Henan Water Resources. 2006 NO.8,p.30-31. 